\title{
Interactive cosmology visualization using the Hubble Ultra Deep Field data in the classroom
}

\author{
Liam J. Nolan ${ }^{1,}$, Mira R. Mechtley ${ }^{1}$, Rogier A. Windhorst ${ }^{1}$, Karen \\ Knierman ${ }^{1}$, Teresa A. Ashcraft ${ }^{1}$, Seth H. Cohen ${ }^{1}$, Scott Tompkins ${ }^{1}$ and \\ Lisa M. Will ${ }^{2}$ \\ ${ }^{1}$ School of Earth and Space Exploration, Arizona State University, Tempe, AZ 85287-1404 and ${ }^{2}$ San Diego City \\ College, San Diego, CA 92101 \\ *Ijnolan@asu.edu
}

\begin{abstract}
We have developed a Java-based teaching tool, "Appreciating Hubble at Hyper-speed" ("AHaH"), intended for use by students and instructors in beginning astronomy and cosmology courses, which we have made available online. This tool lets the user hypothetically traverse the Hubble Ultra Deep Field (HUDF) in three dimensions at over $\sim 500 \times 10^{12}$ times the speed of light, from redshifts $z=0$ today to $z=6$, about 1 Gyr after the Big Bang. Users may also view the Universe in various cosmology configurations and two different geometry modes - standard geometry that includes expansion of the Universe, and a static pseudo-Euclidean geometry for comparison. In this paper we detail the mathematical formulae underlying the functions of this Java application, and provide justification for the use of these particular formulae. These include the manner in which the angular sizes of objects are calculated in various cosmologies, as well as how the application's coordinate system is defined in relativistically expanding cosmologies. We also briefly discuss the methods used to select and prepare the images in the application, the data used to measure the redshifts of the galaxies, and the qualitative implications of the visualization - that is, what exactly users see when they "move" the virtual telescope through the simulation. Finally, we conduct a study of the effectiveness of this teaching tool in the classroom, the results of which show the efficacy of the tool, with over $\sim 90 \%$ approval by students, and provide justification for its further use in a classroom setting.
\end{abstract}

Keywords: Visualization of Relativistic Cosmological Models; Hubble Ultra Deep Field Images; Astronomy Education

\section{Introduction}

In beginning astronomy courses, many non-science majors appear to have a significant lack of understanding - even after taking introductory courses - of basic concepts such as wavelength, the electromagnetic spectrum, the speed of light, lookback time, redshift, and the expansion of the Universe. We believe this lack of concept acquisition or retention represents a significant shortcoming of currently available teaching tools. While pictures, figures, and other static media are certainly effective at communicating many concepts, they tend to be poor at showing these effects in three dimensions, or those that evolve over time (e.g. Sadaghiani 2011 ). Since virtually all cosmological effects require very large time or distance scales to become apparent, a different teaching medium is needed in this case. This is reflected by the well-established need in as- 
tronomy education to support the development of spatial skills, which strongly correlates with performance in all STEM disciplines (Cole et al., 2018).

In addition, the education landscape is changing at a breakneck pace around us, with online learning quickly becoming a preferred option for reasons of convenience, access, and affordability, especially to students of limited means. At its extreme, in the case of large-scale threats to safety, online learning becomes mandatory, as has been so dramatically underlined by the recent outbreak of COVID-19. As millions of students around the world moved from learning in a classroom to learning at home, it became evidently clear that modern classes require new tools for education. Nowhere is this more needed than in the laboratory-type complement to lecture-based learning, and online virtual tools stand to serve this purpose exceptionally well (e.g. Hoeling 2012).

"Appreciating Hubble at Hyper-speed" $(\mathrm{AHaH})$ is an educational tool that aims to address these issues of concept acquisition and retention by providing a visual and interactive learning medium. The project uses data from the Hubble Space Telescope (HST) Cycle 12 Project "GRAPES" (Grism-ACS (Advanced Camera for Surveys) Program for Extragalactic Science; Pirzkal et al., 2004) to build a redshift-sorted database of over 5000 galaxies within the Hubble Ultra Deep Field (HUDF). As a simplified, brief explanation, if one begins Hubble's Law of the Universal Expansion (as per the FLRW model ${ }^{1}$ ),

$$
D=\frac{V}{H_{0}}
$$

and combine with the Doppler approximation as valid for small redshifts $v \approx c z$, one obtains

$$
D=\frac{v}{H_{0}} \approx \frac{c z}{H_{0}}
$$

and can see a galaxy's distance is proportional to redshift, $z$, divided by Hubble's constant, $H_{0}{ }^{2}$. As these galaxies range from redshift $z \approx 0.05$ to $z \approx 6$, their distances span nearly $90 \%$ of the history of the Universe (Yan and Windhorst, 2004; Bouwens et al., 2006; Cohen et al., 2006; Windhorst et al., 2011). Since these data represent the deepest optical images of the Universe ever obtained, they are thus uniquely suited to help students understand the effects of the expanding Universe.

It is worth noting that for the AHaH Application, we developed a custom-balanced RGB version of the original HUDF image to eliminate bright areas being "burned out" and lacking fine detail (Lupton et al., 2004). This custom version is then displayed as semi-transparent, and images are attached to photometric redshifts measured using HyperZ (Bolzonella et al., 2000) using photometry from the publicly available HST optical and near-infrared images (Thompson et al., 2005), as well as spectro-photometric redshifts from Ryan et al. (2007). A comparison of the original STScl color images and our prepared images is shown in Figure 1, and one can see examples of galaxies processed in this way in Figure 2. We explain this in more detail in $\S 14$ (Appendix B).

\footnotetext{
1 For further discussion of the differences between the Hubble's redshiftdistance and velocity-distance laws, see Harrison (1993).

2 We use $H_{0} \approx 68 \mathrm{~km} / \mathrm{s} / \mathrm{Mpc}$ throughout (Planck Collaboration et al., 2018).
}
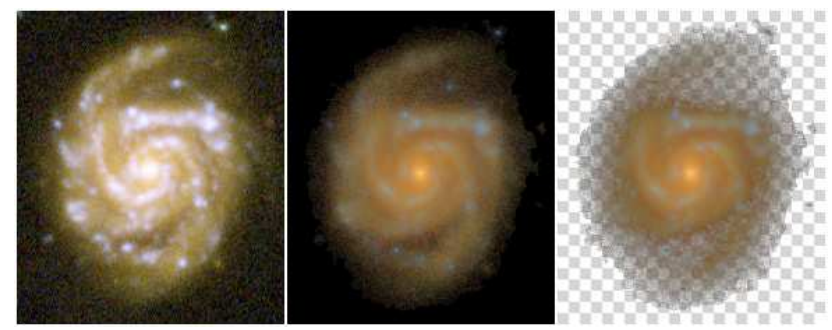

Figure 1. A comparison of three images of HUDF galaxy 7556. The left image is that from the original STSCl release, clearly showing the bright, burned-out knots characteristic of the standard logarithmic image stretch. The center image is our prepared image using the arcsinh stretch described by Lupton et al. (2004), as it appears in the $\mathrm{AHaH}$ application. The right image is our prepared image against an artificially imposed chessboard pattern, showing the included transparency. Note that pixels outside the source are all completely transparent, since they have been removed entirely using the SourceExtractor segmentation map.
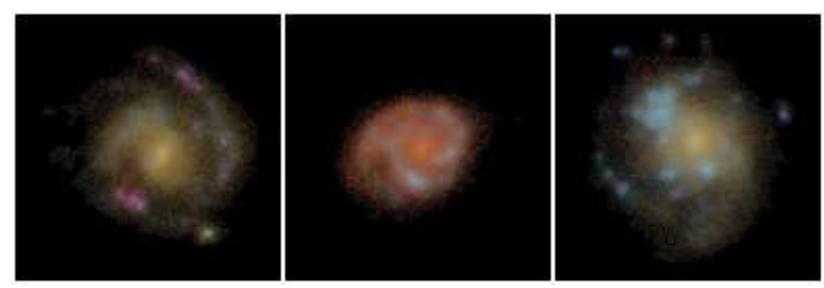

Figure 2. Our prepared images of three galaxies from the HUDF, using the arcsinh stretch described by Lupton et al. (2004). Shown are galaxy 3180 (left), galaxy 5805 (center), and galaxy 6974 (right).

\section{Theoretical Formalism}

As discussed in detail by Wright (2006), there are a number of different methods for calculating distances in cosmology. For our purposes, the most meaningful of these is the comoving radial distance, $D_{R}$, representing the spatial separation of an object and an observer with zero peculiar velocity at a common cosmic time. This distance takes into account the expansion of the Universe, and so is more useful when dealing with distances on very large scales (and thus very large look-back times), as is the case with most galaxies in the HUDF. Henceforth, we shall adopt the convention of referring to the comoving radial distance from Earth to a galaxy as $D_{R}$ in $\mathrm{Gpc}^{3}$, and the comoving coordinate distance between two arbitrary points in the coordinate system as $r_{i j}$.

As we begin to define different cosmological distances, we wish to note that there are many different distance definitions and naming conventions in cosmology which ultimately come down to three distances. For discussions and examples of the variety used in the literature see, for example, Kristian and Sachs (1966), Weinberg (1972), Peebles (1993), Longair (1995), Hogg (1999), Ellis (2007), Etherington (2007), and Ellis (2009). We adopt the names comoving radial distance $\left(D_{R}\right)$, angular size distance $\left(D_{A}\right)$, and luminosity distance $\left(D_{L}\right)$, with $D_{A}$ and $D_{L}$ being related by the "distance duality relation." Regardless of terminology or convenient approximations (i.e. Equation (2)), we carefully use the correct distance formalism "under the hood" to produce the visualization in $\mathrm{AHaH}$.

Returning to the development of the tool, we also wish to calculate the angular sizes of objects as they would be observed from redshifts other than zero. To do so, we need a formula for the angular size distance, $D_{A}$. That is, the distance which satisfies the equation $d \approx \theta D_{A}$ for an object with transverse linear

$31 \mathrm{Gpc}=10^{9} \mathrm{pc}$, and $1 \mathrm{pc}=3.26$ lightyear 
diameter $d$ subtending an angle $\theta$ in the field of view at any redshift in any relativistic cosmology. Following Ribeiro (2005), we note that the correct relation is between solid angle and $R$ over $D_{A}^{2}$, but in the small area of the HUDF we may linearize as previously stated. This is also important to allow the tool to run at a reasonable speed on consumer computers. In a simple Euclidean space, this is the same as the radial distance, but again we must take into account the expansion (and possible curvature) of the Universe, so we must use a separate equation for $D_{A}$ in the $\mathrm{AHaH}$ tool. Details on these definitions and equations are given in $\S 13$ (Appendix A).

In addition, we need to consider how we wish to define the coordinate system for the objects within the Java tool. Although we have very deep HST imaging data that allow us to show how the Universe has changed over time, all of these data were collected at a common time (2003/2004-2014). Moreover, the principal distance measure that we have available, the comoving radial distance $D_{R}$, also assumes a common cosmic time. Thus the most sensible coordinate system is one with three spatial dimensions that makes all calculations for a common cosmic time, viz. when the data were collected. We can then contract the distances in this "comoving coordinate system" as necessary to simulate observations from redshifts greater than zero. The question remains of how we should derive such coordinates from the data that we have in such a way that they will be useful to us - this is discussed in $\S 13.3$ in Appendix A, prior to deriving the equations. We detail our calculations in full in $\S 13$ (Appendix A), which we include for both completeness and instructional purposes - as these calculations should be comprehensible in an intermediate undergraduate-level cosmology course but are not necessary to follow for a demonstration of the tool's educational utility. Ultimately, we are able to develop a relationship between angular size distance and redshift, as well as a coordinate system, which is logical with our available data. We use these relationships (with a few simplifications for computational efficiency) to simulate the "motion" of our $\mathrm{AHaH}$ camera.

\section{Standard Display Mode}

While some might argue that the equations in § 13 (Appendix A) speak for themselves, we believe it is very instructive to consider the qualitative implications to their user - that is, a description of what exactly we see when we "move" the camera in the Java application. For the sake of completeness, we will also detail a number of cosmological effects that have been omitted from the application due to technical limitations. An example of the standard display mode is shown in Figure 3.

When we move the camera to a certain position in the HUDF $(X, Y, Z)$ data cube, we are in general viewing the Universe as it would appear from that point and at that redshift. We must qualify this statement by noting that the simulation accounts only for cosmological effects of changing the camera position no other dynamical, gravitational lensing, evolutionary, or other cosmological or physical effects are simulated. In this sense, $\mathrm{AHaH}$ thus truly, though hypothetically, allows the user to travel through the Universe at "hyper-speed." The highest virtual speed of $\sim 500 \times 10^{12}$ times the speed of light that $\mathrm{AHaH}$ uses to zoom into the HUDF database allows the observer to travel from $z=0$ to $z \approx 6$ in a fraction of a minute, rather than in the $\sim 12.9 \mathrm{Gyr}$ needed if the maximum "travel" speed were really limited to the speed of light $c$, as in the real Universe.

As is covered in many introductory physics and astronomy classes, we use the Euclidean small angle approximation (SAA), where for a fixed object length / and a small angle $\theta$ :

$$
\theta=\tan \left(\frac{l}{D}\right) \approx \frac{1}{D}
$$

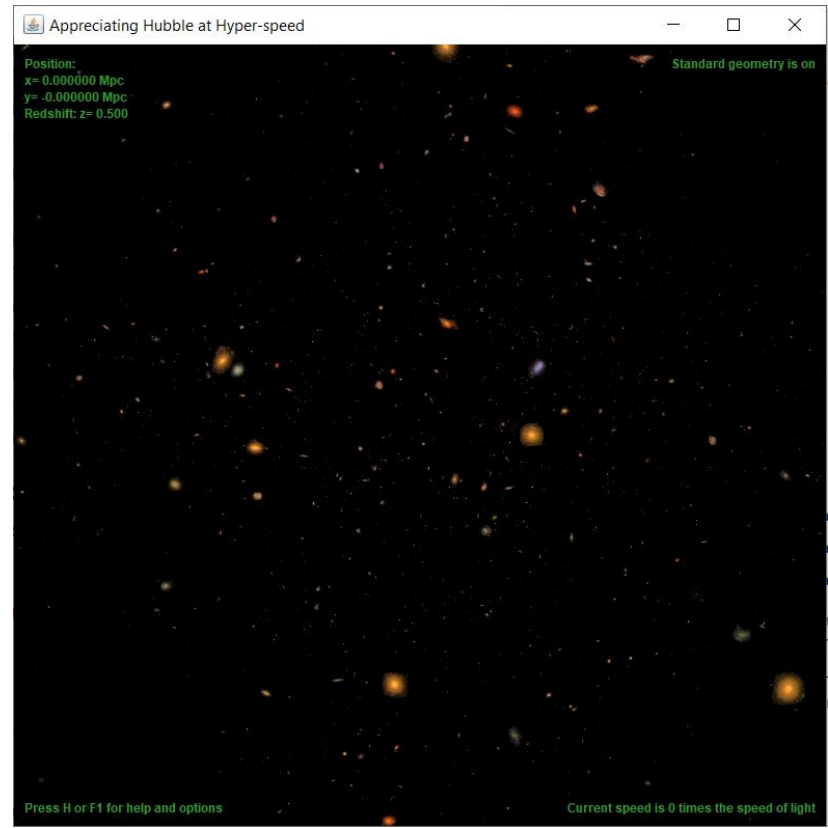

Figure 3. The HUDF data as viewed from redshift $z=0.5$ in the $A H a H$ application, using standard geometry mode, which properly calculates angular sizes. Note how the image is dominated by luminous red early-type galaxies at moderate redshifts of $z \lesssim 1$, where the Universe is older than 6 billion years.

where the distance to the object is $D$. In relativity, we use essentially the same equation, but replace $D$ with $D_{A}$, where $D_{A}$ is a complex function of redshift with a maximum at $z \approx 1.65$. Thus the relativistic SAA is:

$$
\theta \approx \frac{1}{D_{A}}
$$

The somewhat counter intuitive relationship (called the $\Theta$ $z$ relation) between an object's angular size and its redshift in Relativistic Cosmology is readily apparent in the standard display mode. If a user slowly increases the redshift of the camera, high redshift objects will begin to decrease in angular size and move toward the center of the display, eventually reaching a minimum angular size (at redshift $\mathrm{z} \simeq 1.65$ in standard $\Lambda C D M$ cosmology (Planck Collaboration et al., 2018)), and then increasing. Also visible are the effects of galaxy evolution and merging over time. For example, when viewing the Universe from redshift $z=0.5$ as in Figure 3, there are many large spiral and elliptical galaxies visible. Note how the Universe is dominated by luminous red, early-type galaxies at moderate redshifts of $z \approx 1$ in Figure 3. However, when viewing the Universe from redshift $z=1.5$ as in Figure 4, the $\mathrm{AHaH}$ screen is dominated by small and compact blue galaxies. When zooming into the data at $z \geq 1.5$, many of these objects are blue irregular, merging and/or star-forming galaxies. In Figure 4, all red ellipticals of Figure 3 are now "behind us". This Universe at $z \geq 1.5$ is indeed the actively star-forming Universe, i.e., the first 4 billion years after the Big Bang

It should be noted that the application does not make calculations for cosmological surface brightness dimming or changes in color due to redshift or spectral evolution. While certainly feasible to simulate, performing such image manipulation techniques on large numbers of galaxies in real-time is currently too difficult for consumer computers. Moreover, we must also recall that the HUDF data are limited in both magnitude and effective horizon by what could be observed from low Earth orbit. When we view the data from redshifts other than zero, we would expect to see more galaxies overall - includ- 


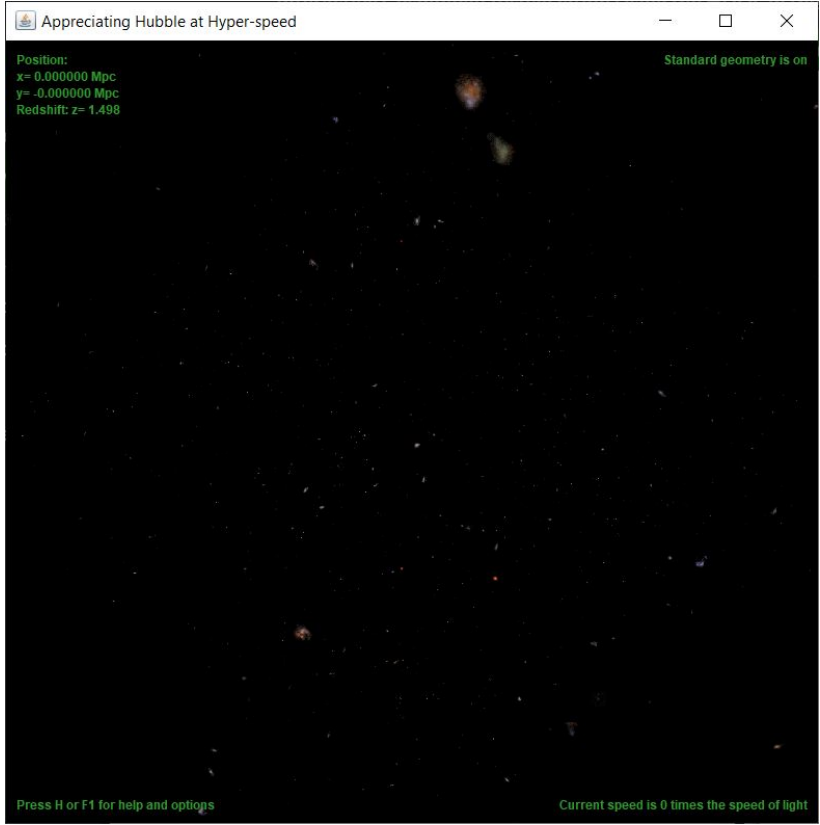

Figure 4. The HUDF data as viewed from redshift $z=1.5$ in the $A H a H$ application, using standard geometry mode. Note how this image is dominated by blue irregular and merging star-forming galaxies, and that all red ellipticals of Figure 3 are now "behind us". This Universe at $z \geq 1.5$ is the actively star-forming Universe, where the Universe is younger than 4 billion years.

ing fainter galaxies - than are present in the current HUDF data. We could choose to simulate these objects as extensions of our data set if desired, but we felt this would not be particularly instructive, and could lead to potential confusion, as fainter galaxies would have to be continuously simulated in increasing numbers by the computer below Hubble's detection limit when traveling from $z=0$ today to $z \approx 6$ ( $\sim$ l Gyr after the Big Bang). Moreover, such simulations have a high degree of uncertainty, and by significantly increasing the size of the data set, would add prohibitively to the computation times. Likewise, we have chosen not to simulate galaxies outside of the original field, which would of course enter the camera's field of view as the user pans around.

\section{Static Geometry Mode}

When a user presses the " $\mathrm{G}$ " key in the AHaH Java tool, they are told that they are viewing the simulation with "unexpanding angular geometry" turned on. What this means specifically is that angular sizes as derived above are no longer affected by the scale factor or curvature of the Universe. After we develop our original coordinates, as in Equation (14), all calculations for angles are simply done with $\theta=\theta_{E}$. This has the visual effect of all galaxies appearing smaller and closer to the center of the viewport (as can be seen in Figure 6 as compared to 5, since all initial angles have been contracted by an expansion factor of ( $1+$ $z$ ) (when the curvature energy density $\Omega_{K}$ is zero; see $\S 5$ for an explanation of the main energy densities at play in Relativistic Cosmology). In this static case, galaxies will also simply increase in angular size as we approach them, as opposed to the angular sizes of high-redshift objects in the real ACDM Universe (Planck Collaboration et al., 2018), which decrease, reach a minimum, and then increase again in angular size as the camera's redshift increases.

This static mode of viewing the simulation has no physical analogue - it is simply meant to convey to the user that there are non-Euclidean aspects of the Universe's geometry, and that the

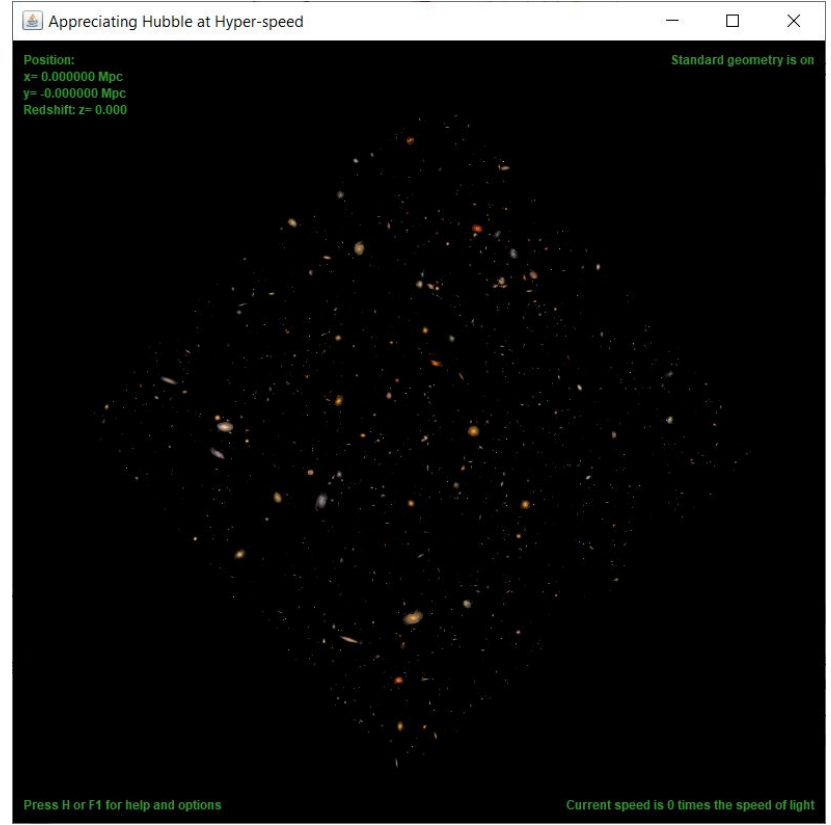

Figure 5. The HUDF data as viewed from redshift $z=O$ in the $A H a H$ application, using standard geometry mode. This displays the entire HUDF, for comparison with Figures 6 and 7 .

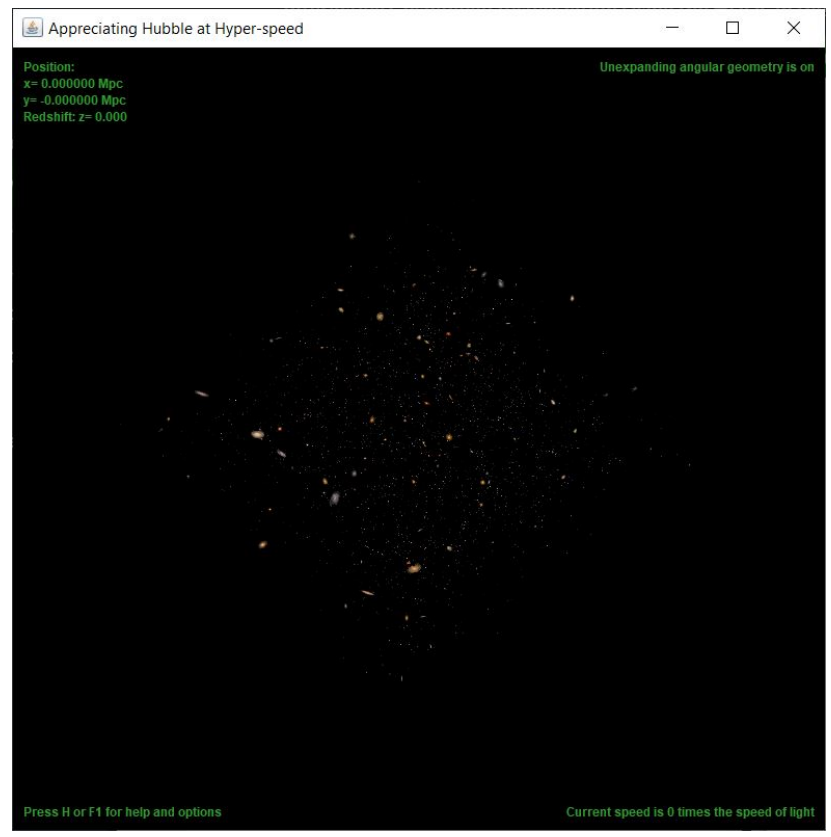

Figure 6. The HUDF data as viewed from redshift $z=0$ in the $A H a H$ application using static geometry mode. This displays the entire HUDF, and one can see the evident "contraction" of the field of galaxies due to the lack of non-Euclidean geometry in the real HUDF data. (That is, the HUDF data in a relativistically expanding cosmology - when shown in Euclidean geometry - compresses all objects towards the image center, since the square cylindrical volume is now not undergoing the expansion, as it should).

angular sizes that we observe in the present have been made larger due to the universal expansion. One should note that this display mode only considers expansion as it relates to angular size - the comoving radial distance is still calculated using the redshift and curvature factors that would not be present in a strictly Euclidean Universe. That is, in the static display mode, we assume that the Hubble Law distance, $D=v / H_{0} \approx\left(c / H_{0}\right) z$, is simply a Euclidean distance unrelated to expansion. This is primarily because our method of calculating the comoving ra- 
dial distance relies using all object redshifts, which is a phenomenon specific only to an expanding Universe, and is therefore the only way we could calculate the distances for all galaxies in other - hypothetical - Universes.

\section{Exploration of Extreme Cosmologies}

One additional capacity of the $\mathrm{AHaH}$ software we wish to note is the representation of wildly different universes from our own, in terms of the cosmological parameters used in the calculations above. As we have already noted, many students have difficulty with relatively esoteric concepts of the energy density parameters of the Universe, and how different portions dominate cosmic behavior over time. To briefly summarize for the reader, $\Omega_{M}$, $\Omega_{R}, \Omega_{\Lambda}$, and $\Omega_{K}$ are the main cosmological parameters, which are the fractions of the Universe's total average energy density that are attributable to matter $(M)$, radiation $(R)$, dark energy $(\Lambda)$, and the curvature of the spatial geometry $(K)$, respectively. It is assumed these are the only relevant contributions to the total energy density $\Omega_{\text {Tot }}$, i.e. $\Omega_{\text {Tot }}=\Omega_{M}+\Omega_{\Lambda}+\Omega_{R}+\Omega_{K}$ (with a spatially flat Universe having $\Omega_{\text {Tot }} \equiv 1$ with $\Omega_{K}=0$ ). However, instead of leaving this to verbal and written descriptions (which can be obtuse), relying on a student's ability to mentally visualize these concepts, $\mathrm{AHaH}$ allows the student to form an approximate image of any cosmology with whatever parameters one could desire. By varying a single parameter, one can see the dramatic (or sometimes less so) impact on our vision of the Universe. We present as an example a massive increase in the vacuum energy density (or Einstein's cosmological constant) $\Omega_{\Lambda}$ from 0.763 to 2.0 (as in Figure 7), which of course results in a Universe rapidly pushed outwards until just a few galaxies hypothetically remain in Hubble's field-of-view. This value was selected because at noticeably higher values of $\Omega_{\Lambda}$, all galaxies disappear nearly completely from view sooner. Of course, just as with the static viewing mode described above, this change in parameters does not produce a perfect representation of a Universe with these parameters, and what is simulated by the software has no physical analogue. However, the changes in display under extreme values of different constants is still instructive as to the effects of these different constants on the structure of the Universe.

\section{Integration in the Classroom}

In order to test the effectiveness of this software in a real education environment, we conducted a study in astronomy classes at Arizona State University in the northern hemisphere fall semester of 2020. It should be noted that due to the outbreak of COVID-19, ASU classes were moved into a partially online Synchronous format for part/all of the northern hemisphere fall 2020 semester, thus introducing an additional variable as compared to a typical semester. This pandemic underlines the necessity of developing online laboratory tools which complement virtual instruction, thus we felt continuing with our study was particularly appropriate.

\subsection{Methods}

We selected the introductory astronomy labs for non-majors (AST 113 northern hemisphere Fall 2020) and for astronomy majors (SES 123 northern hemisphere Fall 2020) for our study in order to focus on the target population mentioned above students first being introduced to this type of astronomy content. Both classes are typically composed of first-year students, so the level of preparation is similar, though students pursuing astronomy majors tend to come into college with more back-

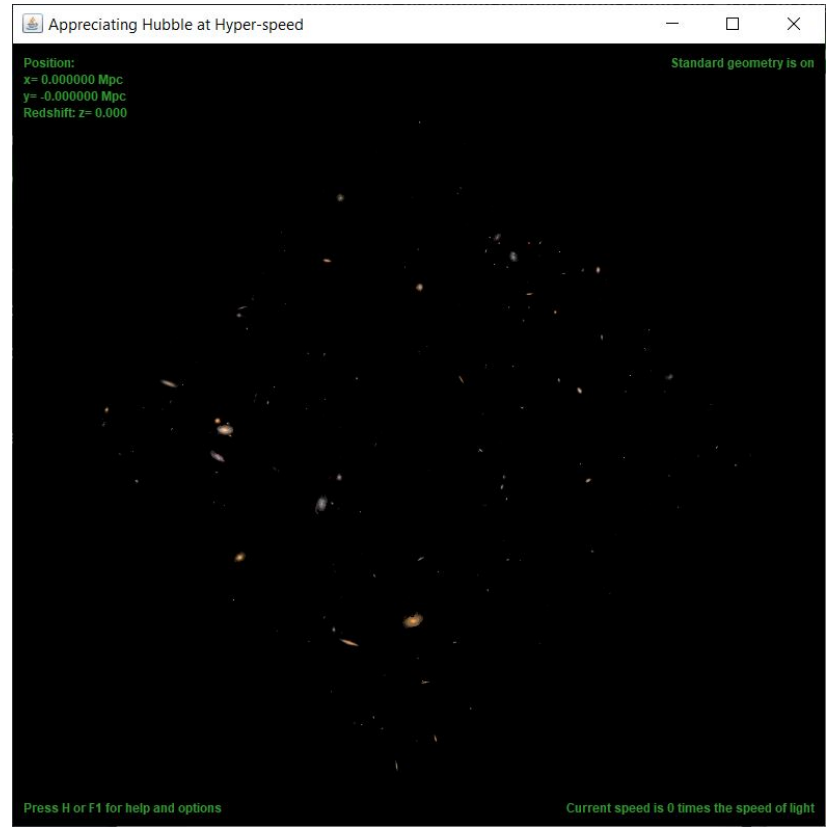

Figure 7. The HUDF data as viewed from redshift $z=0$ in the $A H a H$ application using standard geometry mode, with a vacuum energy density parameter $\Omega \wedge$ value increased from the default value of 0.763 to 2.0. One can see that the extreme value causes most galaxies that are visible in the standard 1 CDM cosmology (Planck Collaboration et al., 2018) to disappear due to vastly increased expansion in this case. This value was selected because noticeably higher values of $\Omega \wedge$ cause all galaxies to disappear entirely - we observers likely live in a relativistic Universe with a "little Lambda."

ground information in our experience. We modified the curriculum of all sections of the lab in two different orders, and compared our results between the two orderings of labs. We requested the ASU Institutional Review Board (IRB) for approval to make this study in the AST 113 and SES 123 Lab classrooms, and their approval, containing all conditions for the study, was filed with ASU. Students who enrolled in these modified lab sections were informed that the new programs with our virtual tool would be available, and that this would not affect the course expectations or standards.

We selected several exercises in the typical class curriculum in both labs for replacement with $\mathrm{AHaH}$ materials based on the advice of the course instructor as to which exercises could have their essential materials folded into other activities or the class lecture component. We then introduced in replacement of these exercises a series of exercises designed by members of our team for $\mathrm{AHaH}$, all of which are available online ${ }^{4}$. Students then conducted these exercises either on AST $113 /$ SES 123 Lab or their own home computers, with the same resources available to them as other activities in class (i.e. technical and subject-matter support from Teaching Assistants, instructor, etc.). Reasonable accommodations for learning and access difficulties as pursuant to University policy were made for, e.g., those students who needed to take this course remotely via the Zoom teleconference platform given their presence elsewhere during the COVID-19 pandemic. In addition, for visually impaired students, we made available the Astronomy Sound of the Month webpage for January $2018^{5}$, which has a full display of the HUDF image and plays a tone when one moves the mouse over a given galaxy, of varying pitch depending on the distance to that galaxy. All lab sections were led for these activities by a combination of Lab Teaching Assistants and the class

4 http://ahah. asu.edu/exercises.htm

5 https://astrosom. com/Jan2018.php 
instructor, as appropriate.

In some cases, material covered by these $\mathrm{AHaH}$ exercises is not part of the subject matter typically evaluated by the course. However, the course instructor believed there was sufficient relation to course content, and students were made aware of this slight alteration. These subjects included, e.g.: galaxy morphology, Hubble's Law, galaxy evolution, the $\Theta-z$ relation, and cosmological parameters.

\subsection{Educational Materials}

The first of the two student activities (referred to as "labs") we led students through in this class was on the subject of galaxy morphology 6 . Students use the $\mathrm{AHaH}$ tool to "fly" through the universe and inspect the morphology of galaxies, either from an instructor-provided list or whichever they find interesting. In the latter case, students are encouraged to pick a variety of galactic morphologies, as they are then asked to classify the galaxies by the Hubble Classification Scheme, and identify various other features. Students then learn more about how different features can lead to conclusions about the populations of stars making up the galaxy. The general learning outcomes produced are:

- Identify galaxies by appearance

- Apply observed colors to the properties of its stars, and

- Understand the differences between the different Hubble classification types.

The second lab was on the subject of "Hubble's Law"7, by which we mean the linear relationship found by Hubble between distance and redshift at low redshifts. In a similar fashion to the galaxy morphology lab, students move through the HUDF to inspect galaxies either from a list or of their choice, but in this case they use the built-in information pop-up on the galaxy to learn its redshift and comoving radial distance, $D_{R}$. Students plot their data points, and then use the "Hubble Law" (the combination of the FLRW model Hubble Law and the Doppler approximation as given in Equation (2)) to calculate $H_{O}$ from the slope of their graphs. A spreadsheet which would work out distances for higher redshifts was made available to students who wished to look at such galaxies. The general learning outcomes produced are:

- Understanding the concept of redshift

- Obtaining and performing calculations with data to produce universal properties (such as $H_{0}$ ), and

- Gaining familiarity with the expansion of the universe.

These goals in particular address an established stumbling point in introductory undergraduate astronomy education - the curvature and behavior of the Universe - as described by Coble et al. (2018).

The goals of these labs are well-described by the Anatomy of Disciplinary Discernment as laid out by Eriksson et al. (2014). For many students, the labs will serve to bring them from the first level, Disciplinary Identification (as students are expected to have been previously informed of terms such as "galaxy" and "redshift"), to the second level, Disciplinary Explanation, and be able to show how galactic properties distribute with redshift. For other students the labs may be able to bring them to the third level, Disciplinary Appreciation, as they grasp the "power" of Hubble's Law and other astronomical relations for astronomers to be able to describe the universe itself from the

6 http://ahah.asu.edu/exercises/galmorph.pdf

7 http://ahah.asu.edu/exercises/hubble.pdf

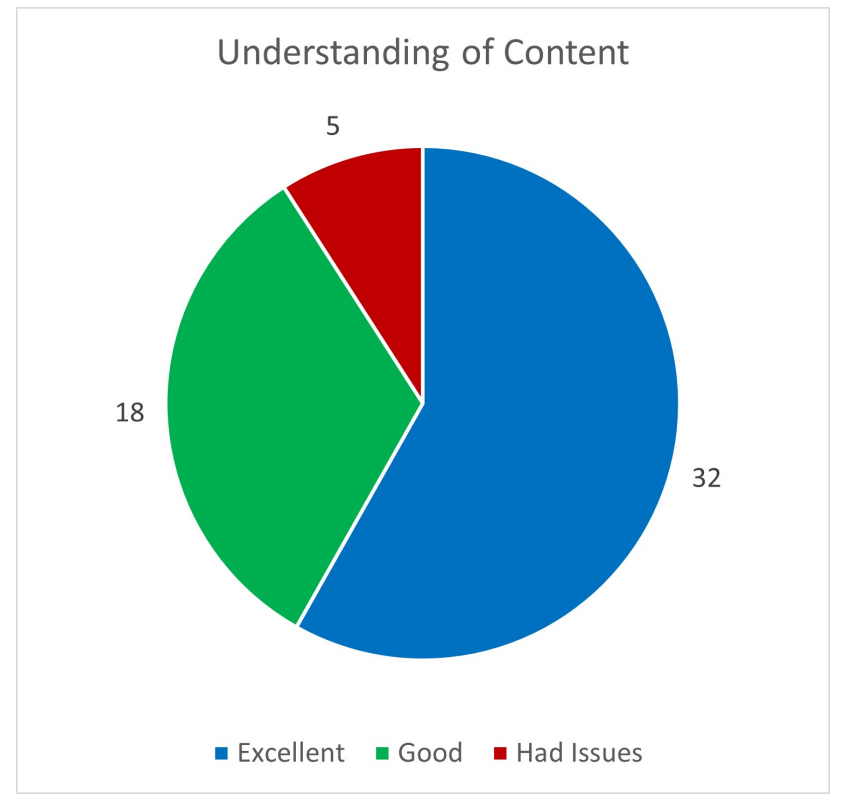

Figure 8. Summary of results from northern hemisphere fall 2020 study, detailed in Table 2, specifically for question on how well the tool helped the students' understanding of the lab content.

trends of galaxies. We also see a few students actually take it upon themselves to learn more about how the standard calculations of the lab are approximations, and how these diverge at high redshifts - thus approaching the fourth and final level, Disciplinary Evaluation. We would also note that a third lab is available on our website concerning galaxy evolution ${ }^{8}$, but was not used in our study.

\subsection{Analysis and Results}

At the end of the northern hemisphere fall 2020 semester class described above, we conducted a survey of students in the enhanced labs for their assessment of the utility of the $\mathrm{AHaH}$ tool, with questions shown in Table 1 , and complete results shown in Figures 8, 9, and 10, and Table 2 for the northern hemisphere fall 2020 class. As can be seen, $90 \%$ of students surveyed thought that $\mathrm{AHaH}$ was good to excellent in helping understanding of lab materials, 92\% thought it was good to excellent in making the lab more interesting, and 91\% thought that it was good to excellent in answering questions they had on the subject matter. Finally, throughout the semester students consistently expressed to instructors a preference for activities with $\mathrm{AHaH}$, as well as greater ease of understanding with the tool. Teaching Assistants regularly reported notably higher amounts of positive feedback on $\mathrm{AHaH}$-related labs as compared to standard lab exercises.

\section{Other Program Uses}

In addition to in the classroom, $\mathrm{AHaH}$ has been and continues to be useful in a wide range of applications in STEM education We have conducted several pilot programs to use this tool in outreach efforts through several events held on and about ASU campus. As in education, while static images have utility in outreach efforts with the general public, people young and old are far more likely to become and stay engaged with dynamic media, such as simulation. As previous studies have shown (e.g. 
Table 1. Questions from survey used in northern hemisphere Fall 2020 AST 113/SES 123 program.

\begin{tabular}{|c|c|c|c|}
\hline \multicolumn{2}{|l|}{ Question } & \multicolumn{2}{|l|}{ Response Type } \\
\hline \multicolumn{2}{|c|}{$\begin{array}{l}\text { I understand that my responses will be used - without my name } \\
\text { and in aggregate - in a journal article, and I consent to this use. }\end{array}$} & \multicolumn{2}{|l|}{ Acknowledgement } \\
\hline \multicolumn{2}{|c|}{$\begin{array}{l}\text { How well did you feel } \mathrm{AHaH} \text { and related labs aided your } \\
\text { understanding of the content covered by these labs? }\end{array}$} & \multicolumn{2}{|c|}{ Multiple Choice on scale of 1 (Poor) to 5 (Excellent) } \\
\hline \multicolumn{2}{|c|}{$\begin{array}{l}\text { How well did you feel } \mathrm{AHaH} \text { and related labs helped in making } \\
\text { labs more interesting? }\end{array}$} & \multicolumn{2}{|c|}{ Multiple Choice on scale of 1 (Poor) to 5 (Excellent) } \\
\hline \multicolumn{2}{|c|}{$\begin{array}{l}\text { How well did you feel } \mathrm{AHaH} \text { and related labs helped answer your } \\
\text { questions about this material? }\end{array}$} & \multicolumn{2}{|c|}{ Multiple Choice on scale of 1 (Poor) to 5 (Excellent) } \\
\hline \multicolumn{2}{|l|}{ Other comments? } & \multicolumn{2}{|l|}{ Free response } \\
\hline Category & Excellent & Good & Had Issues \\
\hline Understanding of Lab Materials & $32(58 \%)$ & $18(32 \%)$ & $5(9 \%)$ \\
\hline Interesting for Lab Exercise & $31(56 \%)$ & $20(36 \%)$ & $4(7 \%)$ \\
\hline Answered Questions & $31(56 \%)$ & $19(35 \%)$ & $5(9 \%)$ \\
\hline Comments & $\begin{array}{l}\text { Excited to use tool as } \\
\text { visualization - many students } \\
\text { were interested in the } \\
\text { differences between galaxies } \\
\text { visually, as well as getting an } \\
\text { idea of differences in distances. }\end{array}$ & $\begin{array}{l}\text { Interface took getting used to - } \\
\text { some students expressed an } \\
\text { interest in the tool being } \\
\text { redesigned as an app, or with } \\
\text { more immediate information } \\
\text { pop-ups. }\end{array}$ & $\begin{array}{l}\text { Common difficulties were with } \\
\text { installation of the tool. }\end{array}$ \\
\hline
\end{tabular}

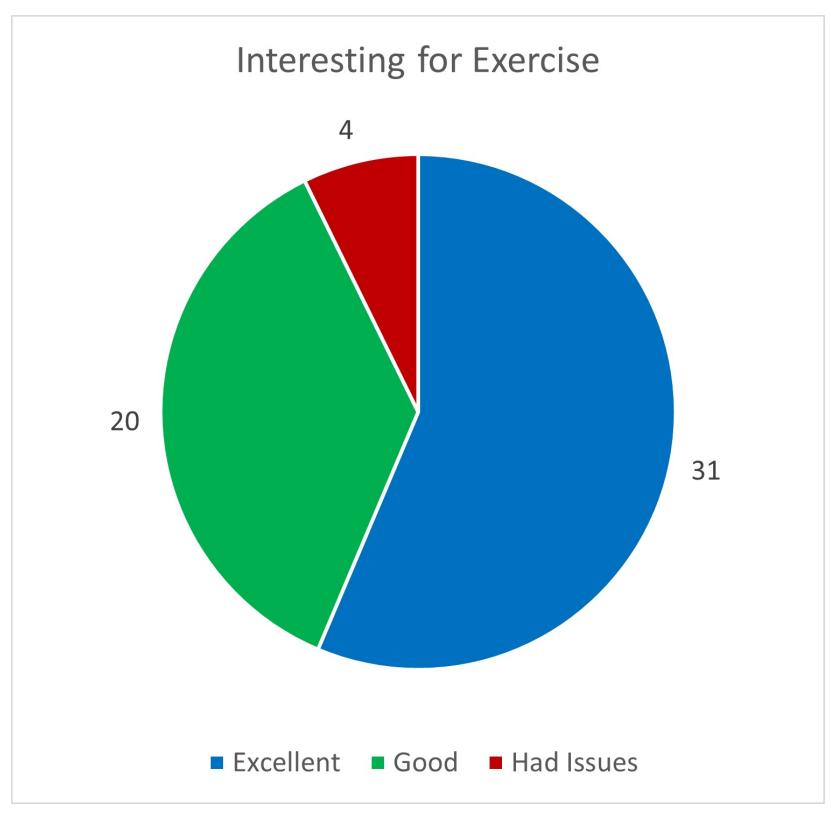

Figure 9. Summary of results from northern hemisphere fall 2020 study, detailed in Table 2, specifically for question on how interesting students found the tool.

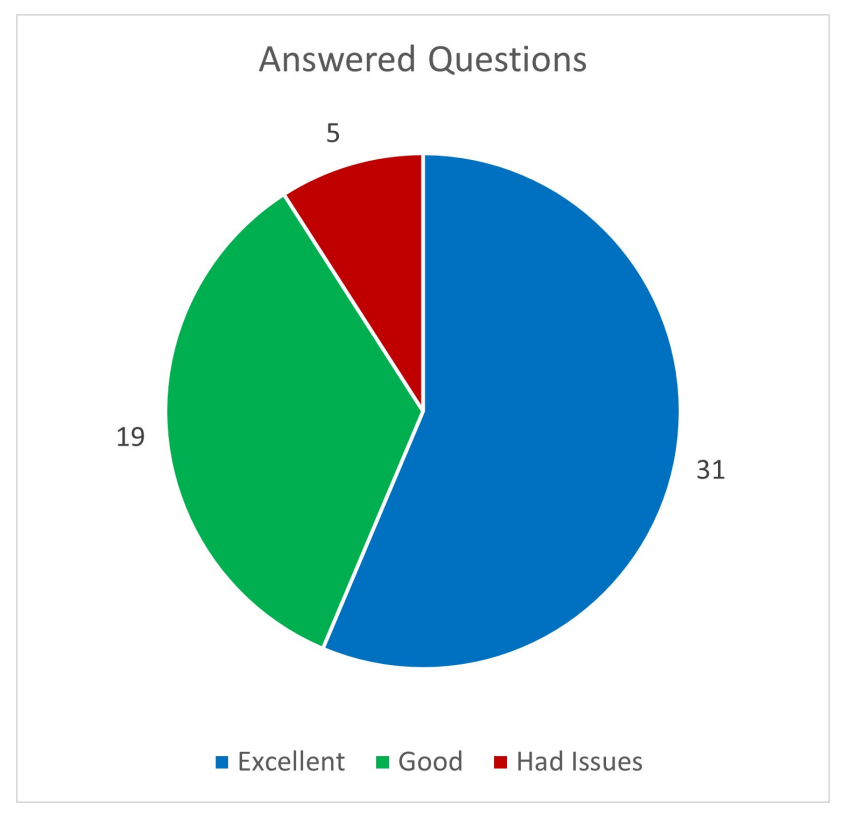

Figure 10. Summary of results from northern hemisphere fall 2020 study, detailed in Table 2, specifically for question on how well the tool answered students' questions. 
Holzinger et al. 2008), simple dynamic media can improve the acquisition and retention of information. In the case of interactive simulation like $\mathrm{AHaH}$, this is likely because the user becomes directly involved in shaping the course of their experience. In our program, this is exemplified by members of the public being able to pick out the galaxies they "fly" towards using $\mathrm{AHaH}$, and can learn more details about these. These programs have shown a qualitative increase in participation by the general public in outreach activities, especially among young children or young adults. As many public educators will attest, half the battle is often getting the public to start using an education opportunity. Thus the use of $\mathrm{AHaH}$ in the classroom is worth consideration by teachers, outreach developers, and organizers.

\section{Conclusion}

We believe that our $\mathrm{AHaH}$ software provides students and instructors with an unique ability to interactively visualize many of the effects of a relativistically expanding Universe, among its other capabilities. The application should help clarify these concepts, and allow students to develop a deeper intuitive understanding of the material. Certain cosmological effects - such as bandpass shifting, $k$-correction, surface brightness dimming, gravitational lensing, and the effects of the magnitude limit and object sizes on the sample completeness limit - have been largely omitted due to computational limitations, but we believe these to be not essential for the understanding of the included effects. For a discussion of these more technical effects, see e.g., Windhorst et al. (2018). In addition, our brief study of the utility of the program in the classroom meets our expectations of its impact on learning, and lends support to our recommendation that further virtual tools be developed in support of online classrooms. We also recommend the use of this tool in other public education and science outreach efforts for its utility in quickly engaging the public.

For the convenience of those who wish to see or modify the particular implementation of the above formulae within the Java software, we have provided source code with the standard distribution of the tool. It is included in the src/ directory of ahah.jar, and may be extracted using the java jar utility or any zlib-compatible de-compressor such as unzip. The tool may be downloaded from the $\mathrm{AHaH}$ website ${ }^{9}$. Further details on $\mathrm{AHaH}$ download instructions and installation are given in the $\mathrm{AHaH}$ user manual, available on the tool website.

\section{Availability of source code and re- quirements}

- Project name: Appreciating Hubble At Hyperspeed

- Project home page: http://ahah.asu.edu

- Operating system(s): Microsoft Windows, Mac OS, or *nix OS

- Programming language: Java

- Other requirements: Java 1.4 .3 or higher, $1 \mathrm{GHz}$ processor, 256 MB RAM, Mouse and Keyboard

- License: BSD-like.

Virtually any modification or redistribution of the application is permitted, with the following caveats:

- Any redistribution must retain the original copyright notice and license file, either with the source code or with the documentation in the case of binary distributions.
- The names of the copyright holders, contributors, and associated institutions may not be used to endorse or promote any derivative works without prior permission.

The application's source code is provided with the standard distribution.

\section{Availability of supporting data and materials}

The data used to develop the teaching tool is available through the sources we cite throughout the paper. As part of our Institutional Review Board agreement, our data may only be published and discussed in aggregate, so we cannot make available our raw survey data.

\section{Declarations}

\subsection{List of abbreviations}

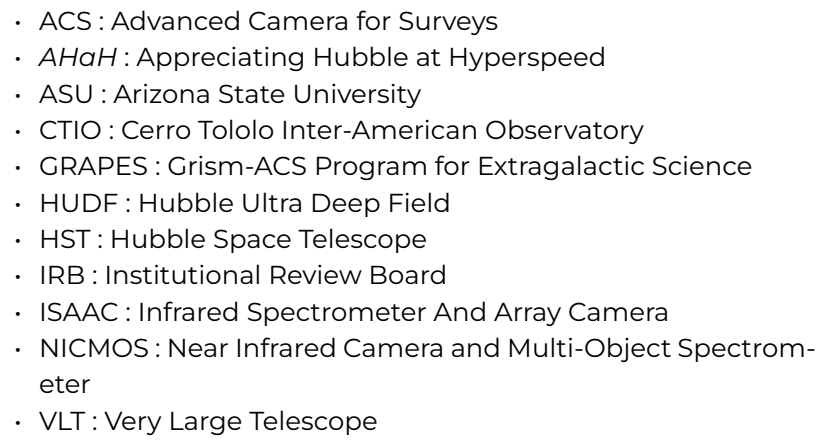

\subsection{Ethical Approval}

As noted above, we filed for and received approval with the ASU Institutional Review Board to conduct our survey in the AST 113 and SES 123 Lab classrooms, and their approval, containing all conditions for the study, was filed with ASU. Students were informed of the new content that would be part of the class, and that there would be a non-graded, optional survey at the end of class concerning the $\mathrm{AHaH}$ tool. All participants in the survey, as indicated in our procedures, gave consent that they were over 18 years of age and that their responses would be reported in aggregate.

\subsection{Consent for publication}

Not applicable, see above.

\subsection{Competing Interests}

The authors declare that they have no competing interests.

\subsection{Funding}

We acknowledge student support from the Arizona State University NASA Space Grant (to LJN and MRM). We acknowledge support from Hubble Space Telescope grants HST-GO10530.07-A, HST-GO-13779.005-A, HST-EO-10530.25-A and HST-EO-13241.001-A from STScl, which is operated by AURA for NASA under contract NAS 5-26555. RAW acknowledges support from NASA JWST Interdisciplinary Scientist grants NAG12460, NNX14AN1OG and 80NSSC18K0200 from GSFC. 


\subsection{Author's Contributions}

LN was the general project lead, developed the survey and other in-classroom components, completed IRB procedures, and wrote the majority of the text on background and program applications. MM led development of the $\mathrm{AHaH}$ code and wrote much of the initial technical paper on the tool's function. RW was in charge of general project management and oversight, as well as geometric formalism, development of related labs, and assisted with paper modification. KK, LW, TA, and RW were in charge of various labs and accommodating the $\mathrm{AHaH}$ survey. $\mathrm{SC}$ helped with the data preparation for the tool, and assisted in paper development. ST gave general project assistance.

\section{Acknowledgements}

We thank Dr. Ned Wright for helpful discussion early in the project. We sincerely appreciate comments from both reviewers of this paper, which helped us better anchor our work in the historical context, and better suit the audience of AEJ and current educational research. We also thank the wonderful Teaching Assistants of AST 113 and SES 123: Angelica Berner, Katherine Elder, Ebraheem Farag, Connor Gelb, Jake Hanson, Isabela Huckabee, Darby Kramer, Kelley Liebst, and Kyle Massingill. Much of our education design was based in part on the work by Hasper et al. (2015).

\section{References}

Beckwith, S. V. W., Stiavelli, M., Koekemoer, A. M., Caldwell, J. A. R., Ferguson, H. C., Hook, R., Lucas, R. A., Bergeron, L. E., Corbin, M., Jogee, S., Panagia, N., Robberto, M., Royle, P., Somerville, R. S., and Sosey, M. (2006). The Hubble Ultra Deep Field. AJ, 132(5):1729-1755.

Bertin, E. and Arnouts, S. (1996). SExtractor: Software for source extraction. AAPS, 117:393-404.

Bertin, E., Mellier, Y., Radovich, M., Missonnier, G., Didelon, P., and Morin, B. (2002). The TERAPIX Pipeline. In Bohlender, D. A., Durand, D., and Handley, T. H., editors, Astronomical Data Analysis Software and Systems XI, volume 281 of Astronomical Society of the Pacific Conference Series, page 228.

Bolzonella, M., Miralles, J. M., and Pelló, R. (2000). Photometric redshifts based on standard SED fitting procedures. $A A P$, 363:476-492.

Bouwens, R. J., Illingworth, G. D., Blakeslee, J. P., and Franx, M. (2006). Galaxies at $z \sim 6$ : The UV Luminosity Function and Luminosity Density from 506 HUDF, HUDF Parallel ACS Field, and GOODS i-Dropouts. APJ, 653(1):53-85.

Coble, K., Conlon, M., and Bailey, J. M. (2018). Investigating undergraduate students' ideas about the curvature of the universe. Phys. Rev. Phys. Educ. Res., 14:010144.

Cohen, S. H., Ryan, R. E., J., Straughn, A. N., Hathi, N. P., Windhorst, R. A., Koekemoer, A. M., Pirzkal, N., Xu, C., Mobasher, B., Malhotra, S., Strolger, L. G., and Rhoads, J. E. (2006). Clues to Active Galactic Nucleus Growth from Optically Variable Objects in the Hubble Ultra Deep Field. APJ, 639(2):731-739.

Cole, M., Cohen, C., Wilhelm, J., and Lindell, R. (2018). Spatial thinking in astronomy education research. Phys. Rev. Phys. Educ. Res., 14:010139.

Dahlen, T., Mobasher, B., Dickinson, M., Ferguson, H. C., Giavalisco, M., Kretchmer, C., and Ravindranath, S. (2007). EvoIution of the Luminosity Function, Star Formation Rate, Morphology, and Size of Star-forming Galaxies Selected at RestFrame 1500 and $2800 \AA$. APJ, 654(1):172-185.

Ellis, G. F. R. (2007). On the definition of distance in general relativity: I. M. H. Etherington (Philosophical Magazine ser.
7, vol. 15, 761 (1933)). General Relativity and Gravitation, 39(7):1047-1052.

Ellis, G. F. R. (2009). Re-publication of: Relativistic cosmology (1971), In: General Relativity and Cosmology, Proc. of the International School of Physics "Enrico Fermi," R.K. Sachs (ed), (New York: Academic Press). General Relativity and Gravitation, 4l(3):581-660.

Eriksson, U., Linder, C., Airey, J., and Redfors, A. (2014). Introducing the anatomy of disciplinary discernment: an example from astronomy. European Journal of Science and Mathematics Education, 2:167-182.

Etherington, I. M. H. (2007). Re-publication of: LX. On the definition of distance in general relativity (1933), Phil. Mag., 15 761. General Relativity and Gravitation, 39(7):1055-1067.

Harrison, E. (1993). The Redshift-Distance and VelocityDistance Laws. APJ, 403:28.

Hasper, E., Windhorst, R., Hedgpeth, T., Van Tuyl, L., Gonzales, A., Martinez, B., Yu, H., Farkas, Z., and Baluch, D. (2015). Methods for Creating and Evaluating 3D Tactile Images to Teach STEM Courses to the Visually Impaired. J. College Sc. Teaching, 44.

Hoeling, B. (2012). Interactive online optics modules for the college physics course. American Journal of Physics, 80:334.

Hogg, D. (1999). Distance measures in cosmology.

Holzinger, A., Kickmeier-Rust, M., and Albert, D. (2008). Modelbased gaussian and non-gaussian clustering. Educational Technology \& Society, 11:279-290.

Kristian, J. and Sachs, R. K. (1966). Observations in Cosmology. $A P J, 143: 379$.

Longair, M. (1995). The deep universe. Saas-Fee Advanced Course23, page 317

Longair, M. (1998). Galaxy formation. New York: Springer.

Lupton, R., Blanton, M. R., Fekete, G., Hogg, D. W., O'Mullane, W., Szalay, A., and Wherry, N. (2004). Preparing Red-Green-Blue Images from CCD Data. PASP, 116(816):133-137.

Peebles, P. J. E. (1993). Principles of physical cosmology. Princeton University Press.

Pirzkal, N., Malhotra, S., Ryan, R. E., Rothberg, B., Grogin, N., Finkelstein, S. L., Koekemoer, A. M., Rhoads, J., Larson, R. L., Christensen, L., Cimatti, A., Ferreras, I., Gardner, J. P., Gronwall, C., Hathi, N. P., Hibon, P., Joshi, B., Kuntschner, H., Meurer G. R., O'Connell, R. W., Oestlin, G., Pasquali, A., Pharo, J., Straughn, A. N., Walsh, J. R., Watson, D., Windhorst, R. A., Zakamska, N. L., and Zirm, A. (2017). FIGS-Faint Infrared Grism Survey: Description and Data Reduction. APJ, 846(1):84.

Pirzkal, N., Xu, C., Malhotra, S., Rhoads, J. E., Koekemoer, A. M., Moustakas, L. A., Walsh, J. R., Windhorst, R. A., Daddi, E., Cimatti, A., Ferguson, H. C., Gardner, J. P., Gronwall, C. Haiman, Z., Kümmel, M., Panagia, N., Pasquali, A., Stiavelli, M., di Serego Alighieri, S., Tsvetanov, Z., Vernet, J., and Yan, H. (2004). GRAPES, Grism Spectroscopy of the Hubble UItra Deep Field: Description and Data Reduction. APJS, 154(2):501-508.

Planck Collaboration, Aghanim, N., Akrami, Y., Ashdown, M., Aumont, J., Baccigalupi, C., Ballardini, M., Banday, A. J., Barreiro, R. B., Bartolo, N., Basak, S., Battye, R., Benabed, K., Bernard, J. P., Bersanelli, M., Bielewicz, P., Bond, J. R., Borrill, J., Bouchet, F. R., Burigana, C., Calabrese, E., Carron, J., Chiang, H. C., Comis, B., Contreras, D., Crill, B. P., Curto, A., Cuttaia, F., de Bernardis, P., de Rosa, A., de Zotti, G., Delabrouille, J., Di Valentino, E., Dickinson, C., Diego, J. M., Doré, O., Ducout, A., Dupac, X., Elsner, F., Enßlin, T. A., Eriksen, H. K., Falgarone, E., Fantaye, Y., Finelli, F., Forastieri, F., Frailis, M., Fraisse, A. A., Franceschi, E., Frolov, A., Galeotta, S., Galli, S., Ganga, K., Gerbino, M., Górski, K. M., Gruppuso, A., Gudmundsson, J. E., Handley, W., Hansen, F. K., Herranz, D., Hivon, E., Huang, Z., Jaffe, A. H., Keihänen, E., Keskitalo, R., Kiiveri, K., Kim, J., Kisner, T. S., Krachmalnicoff, N., Kunz, M., Kurki-Suonio, H., Lamarre, J. M., Lasenby, A., Lattanzi, M., Lawrence, C. R., Le Jeune, M., 
Levrier, F., Liguori, M., Lilje, P. B., Lindholm, V., López-Caniego, M., Lubin, P. M., Ma, Y. Z., Macías-Pérez, J. F., Maggio, G., Maino, D., Mandolesi, N., Mangilli, A., Martin, P. G., Martínez-González, E., Matarrese, S., Mauri, N., McEwen, J. D., Melchiorri, A., Mennella, A., Migliaccio, M., Miville-Deschênes, M. A., Molinari, D., Moneti, A., Montier, L., Morgante, G., Natoli, P., Oxborrow, C. A., Pagano, L., Paoletti, D., Partridge, B., Perdereau, O., Perotto, L., Pettorino, V., Piacentini, F., Plaszczynski, S., Polastri, L., Polenta, G., Rachen, J. P., Racine, B., Reinecke, M., Remazeilles, M., Renzi, A., Rocha, G., Roudier, G., Ruiz-Granados, B., Sandri, M., Savelainen, M., Scott, D., Sirignano, C., Sirri, G., Spencer, L. D., Stanco, L., Sunyaev, R., Tauber, J. A., Tavagnacco, D., Tenti, M., Toffolatti, L., Tomasi, M., Tristram, M., Trombetti, T., Valiviita, J., Van Tent, F., Vielva, P., Villa, F., Vittorio, N., Wandelt, B. D., Wehus, I. K., Zacchei, A., and Zonca, A. (2018). Planck intermediate results. LIII. Detection of velocity dispersion from the kinetic Sunyaev-Zeldovich effect. AAP, 617:A48.

Retzlaff, J., Rosati, P., Dickinson, M., Vandame, B., Rité, C., Nonino, M., Cesarsky, C., and GOODS Team (2010). The Great Observatories Origins Deep Survey. VLT/ISAAC near-infrared imaging of the GOODS-South field. AAP, 511 :A50.

Ribeiro, M. B. (2005). Cosmological distances and fractal statistics of galaxy distribution. A\&A, 429(1):65-74.

Ryan, R. E., J., Hathi, N. P., Cohen, S. H., Malhotra, S., Rhoads, J., Windhorst, R. A., Budavári, T., Pirzkal, N., Xu, C., Panagia, N., Moustakas, L. A., di Serego Alighieri, S., and Yan, H. (2007). The Galaxy Luminosity Function at $z \sim=1$ in the HUDF: Probing the Dwarf Population. APJ, 668(2):839-845.

Ryden, B. (2017). Introduction to Cosmology, 2nd Edition. Cambridge University Press, Cambridge, United Kingdom.

Sadaghiani, H. R. (2011). Using multimedia learning modules in a hybrid-online course in electricity and magnetism. Phys. Rev. ST Phys. Educ. Res., 7:010102.

Thompson, R. I., Illingworth, G., Bouwens, R., Dickinson, M., Eisenstein, D., Fan, X., Franx, M., Riess, A., Rieke, M. J., Schneider, G., Stobie, E., Toft, S., and van Dokkum, P. (2005). The Near-Infrared Camera and Multi-Object Spectrometer Ultra Deep Field: Observations, Data Reduction, and Galaxy Photometry. AJ, 130(1):1-12.

Weinberg, S. (1972). Gravitation and cosmology. Wiley.

Windhorst, R. A., Cohen, S. H., Hathi, N. P., McCarthy, P. J., Ryan, Russell E., J., Yan, H., Baldry, I. K., Driver, S. P., Frogel, J. A., Hill, D. T., Kelvin, L. S., Koekemoer, A. M., Mechtley, M., O'Connell, R. W., Robotham, A. S. G., Rutkowski, M. J., Seibert, M., Straughn, A. N., Tuffs, R. J., Balick, B., Bond, H. E., Bushouse, H., Calzetti, D., Crockett, M., Disney, M. J., Dopita, M. A., Hall, D. N. B., Holtzman, J. A., Kaviraj, S., Kimble, R. A., MacKenty, J. W., Mutchler, M., Paresce, F., Saha, A., Silk, J. I., Trauger, J. T., Walker, A. R., Whitmore, B. C., and Young, E. T. (2011). The Hubble Space Telescope Wide Field Camera 3 Early Release Science Data: Panchromatic Faint Object Counts for 0.2-2 $\mu \mathrm{m}$ Wavelength. APJS, 193(2):27.

Windhorst, R. A., Timmes, F. X., Wyithe, J. S. B., Alpaslan, M., Andrews, S. K., Coe, D., Diego, J. M., Dijkstra, M., Driver, S. P., Kelly, P. L., and Kim, D. (2018). On the Observability of Individual Population III Stars and Their Stellar-mass Black Hole Accretion Disks through Cluster Caustic Transits. APJS, 234(2):41.

Wright, E. L. (2006). A Cosmology Calculator for the World Wide Web. PASP, 118(850):1711-1715.

Yan, H. and Windhorst, R. A. (2004). Candidates of $z \sim=5.5-$ 7 Galaxies in the Hubble Space Telescope Ultra Deep Field. APJL, 612(2):L93-L96.

\section{Appendix A. Derivation of Equations}

Here we include our derivations of the math used in $\mathrm{AHaH}$ at a level appropriate for an intermediate undergraduate cosmology class ${ }^{10}$.

\subsection{Comoving Radial Distance}

To begin, we need the comoving radial distance, $D_{R}$, from the Earth to an object at redshift $z$, derived from the RobertsonWalker metric, as discussed previously, e.g., Longair (Ch. 7 1998), Eqs. 5.33 and 6.13 of Ryden (2017), and Eq. 6 of Wright (2006). We express this as the integral:

$$
D_{R}(z)=\int_{t}^{t_{0}} \frac{c \cdot d t}{a}=\int_{\frac{1}{1+z}}^{1} \frac{c \cdot d a}{a \dot{a}}=\frac{c}{H_{0}} \int_{0}^{z} \frac{d z}{(1+z) \dot{a}}
$$

where the scale factor $a=1 /(1+z)$. The derivative of $a$ with respect to time, $\dot{a}$, is given by the expression:

$$
\dot{a}=\left(\Omega_{M} / a+\Omega_{R} / a^{2}+\Omega_{\Lambda} \cdot a^{2}+\Omega_{K}\right)^{1 / 2},
$$

where $\Omega_{M}, \Omega_{R}, \Omega_{\Lambda}$, and $\Omega_{K}$ are energy density parameters, corresponding to the fractions of the Universe's total average energy density that are attributable to matter $(M)$, radiation (R), dark energy $(\Lambda)$, and the curvature of the spatial geometry $(K)$, respectively. Note that it is assumed these are the only relevant contributions to the total energy density $\Omega_{\text {Tot }}$. That is, we as sume that $\Omega_{\text {Tot }}=\Omega_{M}+\Omega_{\Lambda}+\Omega_{R}+\Omega_{K}$. A spatially flat Universe would have $\Omega_{\text {Tot }} \equiv 1$ with $\Omega_{K}=0$. The default Planck Collaboration et al. (2018) parameters used are: $H_{O}=68 \mathrm{~km} / \mathrm{sec} / \mathrm{Mpc}$, $\Omega_{M}=0.32, \Omega_{\Lambda}=0.68, \Omega_{R}=9 \times 10^{-5}$ with $\Omega_{K}=0$.

We evaluate this integral in steps of 0.05 in $z$ from $z=0$ to $z=20$ to create a look-up table, interpolating linearly to find the value for any arbitrary redshift in between these discrete steps. This is because we must make the calculation frequently and for many objects, so computing the integral manually every time would be computationally prohibitive. The resulting error in this method is generally small enough that it translates to less than one pixel's difference even on high-resolution displays, so this error can safely be ignored for the purposes of the application. We evaluate the integral using the simple midpoint method, which may not be the optimal solution, but was simple to implement and adequately efficient on any home computer. As with the linear interpolation, higher accuracy numerical integration would result in less than one pixel's difference when displayed.

\subsection{Angular Size Distance}

To develop the angular size distance, $D_{A}$, we first need to develop a generalized form of the comoving radial distance $D_{R}$, to express the distance measure to an object at redshift $z_{j}$ as measured by an observer at redshift $z_{i}$. This distance is given by the formula:

$$
D_{R}\left(z_{i}, z_{j}\right)=\left\{\begin{array}{cc}
\mathfrak{R}_{i} \sin \left(r_{i} / \mathfrak{R}_{i}\right) & \text { if } \Omega_{K}<0 \\
r_{i} & \text { if } \Omega_{K}=0 \\
\mathfrak{R}_{i} \sinh \left(r_{i} / \mathfrak{R}_{i}\right) & \text { if } \Omega_{K}>0
\end{array}\right.
$$

where $\mathfrak{R}^{\prime}$ is the radius of curvature of the spatial geometry at redshift $z_{i}$, and $r_{i}$ is the value of the comoving coordinate distance at the same redshift (Longair, 1998; Wright, 2006). These 
correspond to the cases where the spatial geometry of the Universe is spherically curved, flat, and hyperbolically curved, respectively. Recalling that both $r_{i j}^{\prime}$ and $\mathfrak{R}^{\prime}$ scale as $l /\left(1+z_{i}\right)$, and that $\mathfrak{R}=\left(c / H_{0}\right) / \sqrt{\left|\Omega_{K}\right|}$, we next define an intermediate quantity $U$, representing the argument of $\sin$ and $\sinh$ in Equation (7) above:

$$
U=r_{i} / \Re_{i}=r_{0} / \mathfrak{R}_{0}=\left(H_{0} / c\right) \sqrt{\left|\Omega_{K}\right|} r_{0}
$$

We note that since $U$ now depends only upon the cosmology selected by the user and the object's redshift, we may calculate $U$ once per object and re-use it, thus saving CPU time. Using this quantity, we may now rewrite $D_{R}$ as:

$$
D_{R}\left(z_{i}, z_{j}\right)=\frac{\delta(U)}{1+z_{i}} r_{0}
$$

Here, $\delta(U)$ is simply some function of $U$. By substituting $U$ into Equation (7) above, we get the following expression for $\delta(U)$ :

$$
\delta(U)=\left\{\begin{array}{cc}
\frac{\sin (U)}{U} & \text { if } \Omega_{K}<0 \\
1 & \text { if } \Omega_{K}=0 \\
\frac{\sinh (U)}{U} & \text { if } \Omega_{K}>0
\end{array}\right.
$$

Note that $\delta(U)$ expressly depends upon $r_{0}$. The case where $\Omega_{K}=0$ comes from the limit of both $\sin (U) / U$ and $\sinh (U) / U$ as $\Omega_{K} \rightarrow 0$ - one may observe that in this case Equation (9) simplifies to $r_{0} /\left(1+z_{i}\right)$, which is precisely $r_{i}$ as in Equation (7), since as observers we start our $A H a H$ journey at $z_{i}=0$. When we expand with $\mathrm{AHaH}$ into the HUDF galaxy database as it is sorted in redshift, the observer's redshift can take any value $0 \leq z_{i} \leq 20$, although the current set of HUDF postage stamp images does not have any galaxies with $z \geq 6$. We intend to expand this with a future version of $\mathrm{AHaH}$ based on the full data set summarized in Windhorst et al. (2011).

Thus, using our Equation (9) and the equation relating the angular size distance and the distance measure as developed by Longair (1998, Eq. 7.50), the angular size distance from redshift $z_{i}$ to $z_{j}$ is given by:

$$
D_{A}\left(z_{i}, z_{j}\right)=D_{R}\left(z_{i}, z_{j}\right) \frac{1+z_{i}}{1+z_{j}}=\frac{\delta(U)}{1+z_{j}} r_{0}
$$

\subsection{Comoving Coordinate System}

Now that we have developed formulae for $D_{R}$ and $D_{A}$, we can consider the best way to create a coordinate system for the Java application. The data we start with are the redshift $z_{j}$ of object $j$ (with which we can calculate $D_{R}$ ) and four angles: the object's angular size (from the height and width of its image) and the angular separation between the object and the $x$ and $y$ axes, which we define as lines going through the center of the original image. These angles are calculated by taking the corresponding size in pixels and multiplying by the scale in arcsec/pixel of the original HST image ${ }^{11}$.

We would like to use this information to create a coordinate system with the original telescope position at the origin. In a Euclidean space this would present no problem, but we have already remarked that the observed angles are not the same in an expanding Universe as they would be in a Euclidean space. Further, it would be desirable for the Euclidean coordinate dis-

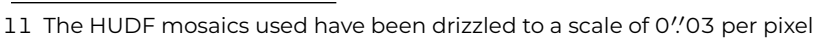

tance to correspond to the comoving radial distance, as this would make calculations significantly simpler. We can accomplish this, but when we create coordinates for each object as such, we need to "correct" the observed angles. That is, we want a "Euclidean angular size" associated with a certain observed angular size. We will call this $\theta_{E}$. An object's angular size is related to its physical transverse diameter, $d$, by the following equation which derives from Equation (11):

$$
d=\theta D_{A}=\theta \frac{\delta(U)}{1+z_{j}} r_{O}=\theta_{E} \frac{1}{1+z_{i}} r_{O}
$$

Note that in the Euclidean case we must contract $r_{0}$ by a factor of $1 /\left(1+z_{i}\right)$ to get the comoving distance from $z_{i}$ to $z_{j}$ as measured by the observer at $z_{i}$ ( $r_{i}$ in Equation (7) above). This is because the proper spatial separation in the current epoch has been stretched by the Universe's expansion, so as seen by an observer at redshift $z_{i}$ it must be scaled appropriately. Hence, the equivalent Euclidean distance between any two points is $D_{E}=r_{0} /\left(1+z_{i}\right)$, in which case we get the Euclidean small-angle approximation back: $d=\theta_{E} D_{E}$.

Thus canceling $r_{O}$, we get the following expression for $\theta_{E}$ from Equation (12):

$$
\theta_{E}=\theta \delta(U) \frac{1+z_{i}}{1+z_{j}}
$$

In our initial data $z_{i}$ is simply zero, so that we create coordinates $(X, Y, Z)$ for an object at redshift $Z$ like:

$$
X=\sin \left(\frac{\delta(U) \theta_{X}}{1+Z}\right) \cos \left(\frac{\delta(U) \theta_{Y}}{1+Z}\right) D_{R}(0, Z)
$$

and similarly for $Y$ and $Z$. We have thus developed a coordinate system of $X, Y$, and $Z$ in comoving $\mathrm{Mpc}$ with the original telescope position at the origin.

\subsection{Simulating Observations From Vantage Points Other Than $z=0$}

Now, when we "move" the Hubble camera virtually to higher redshifts, we do so by moving to some new $\left(X_{c}, Y_{c}, Z_{C}\right)$ value in the coordinate space. (Note that $\mathrm{AHaH}$ does not only "virtually violate the laws of physics" by moving the observer into the HUDF images at $\sim 500 \times 10^{12}$ times the speed of light, but it also has no problem "violating the arrow of time" by allowing the user to move back and forth in redshift through the sorted HUDF image data cube). By construction, the distance measure here is just the Euclidean coordinate distance:

$$
D_{E}=\left(\left(X-X_{C}\right)^{2}+\left(Y-Y_{C}\right)^{2}+\left(Z-Z_{C}\right)^{2}\right)^{1 / 2}
$$

Now to determine where to display an object after we have "moved" the camera, we use the distance calculated with Equation (15) and the Euclidean angular size. Using the redshift of the object of interest, $z_{0}$, and the camera's user-defined redshift, $z_{C}$, we rearrange Equation (13) to get:

$$
\theta=\theta_{E} \frac{1+z_{O}}{\delta_{i j}\left(1+z_{C}\right)}
$$

Here $\delta_{i j}$ follows from Equation (7) and Equation (10) for an object at redshift $z_{j}$ as observed from redshift $z_{i}$. In this case, $\theta_{E}$ is a quantity that we must calculate from our coordinates in the 
usual Euclidean way.

For an object's angular size it is even simpler than for its $(X, Y, Z)$ position, since we do not have to manually calculate $\theta_{E}$. We know that in the Euclidean case:

$$
d=\theta_{O} D_{R}=\theta_{E} D_{E},
$$

where $\theta_{O}$ is the Euclidean angular size from redshift zero, and $D_{E}$ is the coordinate distance from the camera to the object from Equation (15). We then solve for $\theta_{E}$ in Equation (17), $\theta_{E}=\theta_{O} D_{R} / D_{E}$, and substitute back into Equation (16) to obtain an expression for the desired angular size $\theta$ for any object at redshift $z_{O}$ as observed from $z_{C}$ :

$$
\theta=\theta_{O}\left(\frac{D_{R}}{D_{E}}\right) \frac{1+z_{\circ}}{\delta_{i j}\left(1+z_{C}\right)}
$$

\section{Appendix B. Specialized Data Prepa- ration}

To develop the images used in $\mathrm{AHaH}$ we first created a custombalanced RGB version of the HUDF image ${ }^{12}$. While the image provided in the original press releases ${ }^{12}$ would have been adequate, it has the undesirable characteristic that very bright areas, such as bulges in large spiral galaxies, appear burned-out and lack fine detail. The HUDF data used was taken to roughly equal depths in the $B-, V-, i^{\prime}$-, and $z^{\prime}$-band filters, which have central wavelengths of $\sim 320$ (Blue), 5920 (Visual), 7690 (Red), and $9030 \AA$ (near-Infrared), respectively (Beckwith et al., 2006), so we created a three-channel color image by first combining the $B$ - and $V$-bands, applying weights based on the sky signalto-noise ratio ${ }^{13}$. We then used the algorithm developed by Lupton et al. (2004) to create the combined RGB image, with the combined $B+V$-bands as the blue channel, the $i^{\prime}$-band as the green channel, and the $z^{\prime}$-band as the red channel ${ }^{14}$. Besides showing more detail in bright areas, this method has the added benefit that an object with a specified astronomical color has a unique color in the composite RGB image. A comparison of the original STSCl color images and our prepared images is shown in Figure 1. The full HUDF image using this color preparation technique is also available as an interactive map online ${ }^{15}$. One can see examples of galaxies processed in this way in Figure 2.

The galaxies represented in the $\mathrm{AHaH}$ application were $i^{\prime}$ band $(8000 \AA)$ selected using the SourceExtractor algorithm (Bertin and Arnouts, 1996; Bertin et al., 2002) with a detection threshold of $1.8 \times$ the rms noise level $(1.8 \sigma)$ above the local sky. The $i^{\prime}$-band (at $z \approx 6$ ) dropouts of Yan and Windhorst (2004) were added by hand. We then created color JPEG "stamp" images for each individual object, using the SourceExtractorgenerated segmentation map to mask as black any pixels outside the detected source. These "stamps" were then converted pixel-for-pixel to PNG images, which employ a lossless compression algorithm - no image quality was thus lost. We then developed a transparency map based on each pixel's brightness,

12 See http://imgsrc.hubblesite.org/hu/db/2004/07/images/a/formats/ full_jpg.jpg for the full-resolution $60 \mathrm{Mb}$ HUDF ACS image in the BViz filters, and https://www.asu.edu/clas/hst/www/aas2014/ HUDF14-pan-UVrendered.jpg for the deepest 13-filter panchromatic 841-orbit Hubble image.

13 We applied a weight of 0.765 in $V$ and 0.235 in $B$ to balance the image depths. For details, see Windhorst et al. (2011)

14 The channels were first scaled proportional to the data zero points - Red: 716.474, Green: 345.462, Blue: 254.449, see https://hst-docs.stsci. edu/acsihb.

15 http://ahah.asu.edu/clickonHUDF/index.html which was saved into the PNG alpha channel ${ }^{16}$. The resulting images can thus be displayed as semi-transparent, allowing objects in the distance to show through the dim regions of objects in the foreground, as is also possible in the real Universe.

Photometric redshifts for the galaxies were measured with the HyperZ package (Bolzonella et al., 2000), using a combination of the original HST-ACS four-band $\left(B V i^{\prime} z^{\prime}\right)$ data from the HUDF, along with $Y$-, J- and $H$-band near-IR data from HST NICMOS (Near Infrared Camera and Multi-Object Spectrometer) (Thompson et al., 2005). We have supplemented the photometric redshifts with spectro-photometric redshifts measured by Ryan et al. (2007), which incorporate the aforementioned $B V i^{\prime} z^{\prime} J H$ data, as well as grism spectra from GRAPES (Pirzkal et al., 2004, 2017), the U-band observations from CTIO (Cerro Tololo Inter-American Observatory) Mosaic II (Dahlen et al., 2007), and $K_{S}$-band data from VLT-ISAAC (Very Large Telescope - Infrared Spectrometer And Array Camera, e.g. Retzlaff et al. (2010)). For a summary of all these data and the quality of the spectro-photometric redshifts, see Ryan et al. (2007). When available, we have chosen to use the more reliable spectrophotometric redshifts.
16 The alpha channel of a PNG contains the "transparency" of each pixel, which is more straightforwardly the opacity of the pixel. For more, see the PNG specification page from W3: https : //www .w3.org/TR/PNG-DataRep. html. 